\title{
PLANTÍO DE IGLESIAS E INCREMENTO DE FELIGRESES ADVENTISTAS EN LA ZONA METROPOLITANA DE EUNAPOLIS, BAHÍA, BRASIL, ENTRE LOS AÑOS 1980 Y 2010
}

Vander Lopes, Heraldo Amazonia Adventist College heraldo.lopes@faama.edu.br

El crecimiento de la Iglesia Adventista del Séptimo Día a nivel mundial, ha sido explosivo en las últimas dos décadas. El objetivo de esta investigación es describir cómo la estrategia del plantío de iglesias, contribuyó al incremento de feligreses adventistas en la zona metropolitana de Eunapolis, Bahia, Brasil. Los resultados indican que entre 1980 y 2010 la Iglesia Adventista de Eunápolis creció continuamente, superando los indices de crecimiento de la Iglesia Adventista en el territorio de Brasil. El número de iglesias adventistas en Brasil creció en 430,60\% en ese periodo; en Eunapolis aumentó en 1,675\%. En relación a los miembros, Brasil aumentó en $366,88 \%$ en ese periodo mientras que Eunapolis creció en 1.601,77\%. Por lo tanto se concluye que la estrategia: Plantio de Iglesias, practicada en Eunapolis es efectiva y mantiene un crecimiento constante.

Palabras clave: Plantío de iglesias, incremento de feligreses, crecimiento de iglesia 
Summary: The growth of the Seventh-day Adventist Church worldwide has been explosive in the last two decades. The objective of this research is to describe how the church planting strategy contributed to the increased Adventist Church members in the metropolitan area of Eunapolis, Bahia, Brazil. The results indicate that between 1980 and $2010 \mathrm{Eu}$ nápolis Adventist Church grew continuously surpassing the growth rates of the Adventist Church in the territory of Brazil. The number of Adventist churches in Brazil grew by $430.60 \%$ in that period, while in Eunapolis it increased by 1,675\%. In relation to the members, Brazil increased by $366.88 \%$ in that period while Eunapolis grew by $1601.77 \%$. It is therefore concluded that the strategy of planting churches practiced in Eunapolis is effective and produces a constant growth.

Palabras clave: Church planting, Membership growth 


\section{Introducción}

El plantío de iglesias ha sido bien estudiado y publicado. ${ }^{1}$ Incluso la Iglesia Adventista del Séptimo Día (IASD) tiene una página oficial dedicada al fomento del mismo en el territorio de América del Sur. ${ }^{2}$ Según Wagner, "la metodología evangelística más eficaz, bajo el cielo, es la

1 Véase, por ejemplo: David J. Hesselgrave, Planting Churches Cross-Culturally: A Guide for Home and Foreign Missions (Grand Rapids, MI: Baker, 1980); Ronaldo Lidório, Plantando Igrejas (São Paulo: Cultura Cristã, 2007); Aubrey Malphurs, 10 Planting Growing Churches for the 21 Century: A Comprehensive Guide for New Churches and Those Desiring Revival (Grand Rapids, MI: Baker, 1998); C. Peter Wagner, Church Planting for a Greater Harvest (Ventura, CA: Regal Books, 1990); Roger Dudley and Clarence Gruesbeck, Plant a Church, Reap a Harvest (Boise, ID: Pacific Press, 1989); Roland Allen, Missionary Methods, St. Paul's or Ours? (Grand Rapids, MI: Wm. B. Eerdmans, 1962); Talmadge Amberson, Birth of Church: A Biblical Basis for Church Planting (Nashville, TN: Broadman, 1979); David J. Hesselgrave, Plantar Igrejas (São Paulo: Vida Nova, 1988); Larry L. Lewis, The Church Planter's Handbook (Nashville, TN: Broadman, 1993).

2 Divisão Sul Americana (DSA), "O Projeto - Plantío de Igrejas", Plantío de Igrejas, http://plantíodeigrejas.com.br/o-projeto/ (consultado: 25 de julio, 2012). plantación de nuevas iglesias". ${ }^{3}$ Elena G. de White dice: "junto a todas las aguas han de sembrar la simiente de verdad, visitando un lugar tras otro para suscitar iglesia tras iglesia”. ${ }^{4}$

La ciudad de Eunapolis, situada en el extremo sur del estado de Bahía, nordeste del Brasil, por reunir ciertas características en cuanto al crecimiento de la iglesia, ha sido elegida para realizar esta investigación. Actualmente la economía está basada en el comercio, agricultura, ganadería, servicios, turismo y la producción de papaya. ${ }^{5}$

Con la explotación de madera en la región, en la segunda mitad de la década de los años

3 C. Peter Wagner, Plantar Igrejas para a Grande Colheita (São Paulo: ABBA, 1993), 11.

4 Elena G. de White, Testimonios para la iglesia (Doral, FL: Publicaciones Interamericanas, 2003), 23.

5 Instituto Brasileiro de Geografia e Estatística (IBGE), "Produto Interno Bruto dos Municípios 2004-2008”, s.d., http:// www.ibge.gov.br/home/estatistica/economia/ pibmunicipios/2004_2008/tabelas_pdf/tab01.pdf (consultado: 22 de julio, 2012). 
70, numerosos capixabas ${ }^{6}$ vinieron a vivir y trabajar. La mayoría de ellos eran descendientes de los luteranos alemanes que en el siglo XIX llegaron al estado de Espíritu Santo, con el fin de cuidar las plantaciones de café.

\section{Llegaron al Brasil para que-} darse permanentemente. No tenían planes de regresar a Alemania, por lo que se dedicaron a trabajar. Debido a sus antiguas tradiciones cristianas, para los capixabas, las reuniones religiosas deben ser realizadas en templos.?

6 Según los estudiosos de Tupi, capixaba significa limpieza, cortar el césped, tierra limpia para la siembra. Los indios que vivían allí llamaban capixaba al maíz y la yuca. Por lo tanto, la población de Victoria pasó a llamar de capixabas a los indios que habitaban la región, luego este nombre designó a todos los habitantes del Estado de Espíritu Santo. Ver: Governo do Estado do Espírito Santo, "A Origem do Termo Capixaba", Portal do Governo do Estado do Espírito Santo, http://www.es.gov.br/ EspiritoSanto/ paginas/origem_do_termo_capixaba.aspx (consultado: 22 de julio, 2012).

7 Governo do Estado do Espírito Santo, "Presença Européia", Portal do Governo do Estado do Espírito Santo, http:// www.es.gov.br/EspiritoSanto/paginas/pre-
El mensaje adventista llegó a esta ciudad en 1956. El crecimiento de la Iglesia fue lento hasta la década de los 70'. La Iglesia Central de Eunapolis se organizó en 1974, con 65 miembros. ${ }^{8}$ En 1978, se fundó la segunda Iglesia, el ABBI. La Iglesia del barrio de Gusmão fue fundada en 1979 y de Juca Rosa en 1980.9 Desde entonces, la cantidad de iglesias establecidas y de miembros, ha crecido sostenidamente, llegando a 75 congregaciones con 7081 miembros, organizados en 8 distritos pastorales. ${ }^{10}$

Por lo tanto, con la capacidad emprendedora, recursos financieros y su cultura religiosa de

senca_europeia.aspx (consultado: $22 \mathrm{de}$ julio, 2012).

8 Alaíde Sacramento, "Entrevista Sobre a História da Igreja Adventista em Eunapolis", entrevista por Heraldo Vander Lopes, 3 de mayo, 1996.

9 Jair Passos, "Entrevista Sobre a História da Igreja Adventista em Eunapolis", entrevista por Heraldo Vander Lopes, 4 de mayo, 1996.

10 Secretaria da Associação Bahia Sul (ABS), Distritos e Igrejas em 31/12/2010 (Itabuna. BA: Associação Bahía Sul da IASD, fevereiro, 2011). 
construir iglesias para las reuniones de adoración a Dios, varios templos comenzaron a construirse en la sede y después en la zona metropolitana de Eunapolis. ${ }^{11}$ Entre 1980 y 2010, la población de Brasil pasó de 121150573 a 190755799 habitantes, creciendo así, en un $57,45 \%$.

En este mismo período, la población de Eunapolis pasó de 90795 a 253389 habitantes, creciendo un $179,07 \%$.

Entre 1980 y 2010, los adven12 tistas en Brasil aumentaron de 280664 miembros a 1267 738, es decir, en un 351,69\%. En esta época, los adventistas, en el área metropolitana de Eunapolis, crecieron de 561 a 6705 miembros. Un aumento de 1095,18\%. Entre 1980 y 2010, el número de iglesias adventistas en Brasil creció de 3055 a 13610 iglesias, es decir, se incrementó en $345,49 \%$.

11 Secretaria da Associação Bahia Sul (ABS), Data da Fundação da Igreja, Quantidade de Igrejas e Membros até 31/12/2011 - Região de Eunápolis (Itabuna. BA: Associação Bahia Sul da IASD, julio 2012).
Durante ese tiempo, el número de iglesias adventistas en la zona metropolitana de Eunapolis aumentó de 7 a 71 . Un incremento del 914,28\%.

Por lo tanto, la tasa de crecimiento en el número de iglesias y miembros en el área metropolitana de Eunapolis, fue mayor que la tasa de crecimiento de la población en Brasil y de la Iglesia Adventista del Séptimo Día en el país, durante el mismo período.

\section{Antecedentes de la investigación}

No se encontraron estudios disponibles que mencionen el crecimiento de las Iglesias adventistas en la ciudad de Eunapolis. La literatura que se menciona a continuación tiene que ver con el plantío de Iglesias en otros contextos, pero sus principios se pueden relacionar con lo que sucedió en Eunapolis.

La literatura sobre el plantío de Iglesias es muy amplia, por ejemplo Multihousing Congre- 
gations: How to Start and Grow Christian Congregations in Multihousing Communities, de Bunch, Kneisel y Oden; ${ }^{12}$ Church Planting For a Greater Harvest, de C. Peter Wagner; ${ }^{13}$ The Purpose Driven Church, de Rick Warren; ${ }^{14}$ Plant a Church, Reap a Harvest, de Dudley y Gruesbeck ${ }^{15}$ son ejemplos clásicos de literatura sobre crecimiento de iglesia. Plantando Igrejas, de Ronaldo Lidório, presenta la teoría y la práctica del

12 David Bunch, Jarvey Kneisel, and Barbara Oden, Multihousing Congregations: How to Start and Grow Congregations in Multihousing Communities (Atlanta, GA: Smith Publishing, 1991); Hesselgrave, Planting Churches; Paul G. Hiebert and Eloise Hiebert Meneses, Incarnational Ministry: Planting Churchs in Band, Tribal, Pleasant and Urban Societies (Grand Rapids, MI: Baker, 1995), Robert E. Logan, Beyond Church Growth (Old Tapan, NJ: Fleming H. Revell, 1989); David W. Shenk y Ervin R. Stutzman, Creating Communities of the Kingdom: New Testament Models of Church Planting (Scotttdale, PA: Herald, 1988).

13 Wagner, Church Planting for a Greater Harvest; Wagner, Plantar Igrejas para a Grande Colheita.

14 Rick Warren, The Purpose Driven Church: Growth Without Compromising Your Message and Mission (Grand Rapids, MI: Zondervan, 2007).

15 Dudley and Gruesbeck, Plant a Church, Reap a Harvest. proceso, además brinda apoyo a través de internet, con cursos, conferencias y materiales. ${ }^{16}$

Crecimiento: claves para revolucionar su iglesia, de Daniel e Isabel Rode, se basó en las ideas de expertos de crecimiento de la Iglesia, como Peter Wagner, Rick Warren y Christian Schwarz ${ }^{17}$ Guia de Plantio de Igrejas, de Emílio Abdala, es un trabajo que ha llenado el vacío existente en la literatura adventista, respecto al plantío de iglesias, especialmente en Brasil. ${ }^{18}$

16 Lidório, Plantando Igrejas; Ronaldo Lidório, "O que define a plantação de igrejas, teológica e biblicamente ?", Instituto Antropos - Pesquisa Social e Missiologia Aplicada, http://instituto.antropos.com.br/v3/index. php?option $=$ com_content $\&$ view $=$ article\&id=613:o-que-define-a-plantacao-de-igrejas-teologica-e-biblicamente- $\&$ catid $=42$ : consultoria-online-em-plantío-de-igrejas\&Itemid $=58$ (consultado: 25 de julio, 2012); Ronaldo Lidório, "Ronaldo e Rossana Lidório - Teologia bíblica da contextualização", http://www.ronaldo.lidorio.com.br/index. php?option $=$ com_content\&task=view\&id= 63\&Itemid=26 (consultado: 25 de julio, 2012).

17 Daniel Rode e Isabel Rode, Crescimento: chaves para revolucionar a sua igreja (Engenheiro Coelho, SP: UNASPRESS, 2007).

18 Emílio Abdala, Guia de Plantío de 


\section{Problema y objetivo general}

Por lo descrito anteriormente, se formuló la siguiente pregunta que expresa tanto el problema como el objetivo de esta investigación: ¿Cómo la estrategia del plantío de iglesias contribuyó al incremento de feligreses adventistas en la zona metropolitana de Eunapolis, Bahía, Brasil, entre los años 1980 y 2010?

\section{Marco bíblico-teológico del plantío de iglesias}

En el Antiguo Testamento la evangelización o la misión se centró en el lugar de culto, el Santuario (Ex 25:8; Sal 134:2). Las personas venían al santuario para adorar a Dios (Sal 68:29; 2 Cr 20:8-9). El pueblo de Israel era una nación sacerdotal que experimentaba en su vida el poder de Dios (Ex 19:6). Las naciones venían a este lugar y recibían

Igrejas (Artur Nogueira, SP: UCB, 2010). las instrucciones del pueblo elegido, descendientes de Abraham, Isaac y Jacob (1 Re 10:1-10; Is 60:1-7). Este modelo misional era centrípeto. ${ }^{19}$

En el Nuevo Testamento el modelo se invierte, convirtiéndose en centrífugo. Los testigos van a personas de todas las naciones, tribus, lenguas y pueblos, y donde se encuentran, establecen lugares de culto abiertos a todos (Mt 28:18-20). ${ }^{20}$

19 Sobre la misión en el Antiguo Testamento, ver: David J. Bosch, Missão Transformadora: mudanças de paradigma na teologia da missão (São Leopoldo, RS: Editora Sinodal, 2002), 37; Timóteo Carriker, A Visão Missionária da Bíblia: uma história de amor (Viçosa, MG: Ultimato, 2005), 20; Johannes Blauw, A Natureza Missionária da Igreja: exame da teologia bíblica da missão (São Paulo, SP: ASTE, 1966), 36.

20 David J. Bosch, in The Structure of Mission: An Exposition of Matthew 28:1-20. (Grand Rapids, MI: Wm. B. Eerdmans, 1983), 35; Bosch, Missão Transformadora, 37-39; René Padilla, "Mensaje inaugural - todo el evangelio para todos los pueblos desde América latina", ed. CLADE III: Tercer Congreso latinoamericano de Evangelización, Quito, 24 de agosto a 4 de septiembre de 1992 (Quito: Fraternidad Teológica latinoamerina, 1992), 14; René Padilla, O Que é Missão Integral? (Viçosa, MG: Ultimato, 2009). 
La idea de la misión está vinculada al concepto de Missio Dei, ya que no hay una misión de envío fuera de Dios y todo lo que la iglesia hace se deriva de la acción de enviar de Dios: Padre, Hijo y Espíritu Santo. ${ }^{21}$

Por lo tanto, misión y Missio Dei son la misma acción, sin embargo, misión utiliza el término para referirse a la missio ecclesiae (misión de la iglesia). Este efecto solo se hace real cuando se entiende que la iglesia fue encargada por un Dios uno y trino, como afirma J. Verkuyl:

21 Aguinaldo Leônidas Guimarães, "Compreendendo e Definindo os Termos Relacionados à Missão”, Missão Urbana, s.d., http://missao-urbana.com/ artigos/compreendendo-definindo-termos-relacionados-a-missao/(consultado: 30 de julio, 2012); J.A. McIntosh, "Missio Dei", in Evangelical Dictionary of World Missions (Grand Rapids, MI: Baker, 2000), 631-632; Bosch, Missão Transformadora, 637; A.S. Moreau, Mission and Missions, Evangelical Dictionary of World Missions (Grand Rapids, MI: Baker, 2000), 636-640; Bosch, Missão Transformadora, 467; Jürgen Moltmann, The Church in the Power of the Spirit: A contribution to messianic ecclesiology (London, UK: SCM, 1977), 64.

\begin{abstract}
"Dios el Padre envió al Hijo y el Hijo es el enviado y enviador. En conjunto, el Padre y el Hijo envian al Espiritu Santo, que, a su vez, envía a la iglesia, las congregaciones, los apóstoles y siervos, poniendo en ellos la obligación de hacer su trabajo". 22
\end{abstract}

La missio ecclesiae es simplemente la Missio Dei. "Dios no tiene una misión para su iglesia, sino una iglesia para su misión”. Aquí se puede poner de relieve otro énfasis que surgió en la conferencia de Willingen y más tarde en una conferencia en la Ciudad de México, en la que se relacionó la Missio Dei con la missio ecclesiae, afirmando que "no hay participación en Cristo sin la participación en su misión”.23

22 Bosch, Missão Transformadora, 17; Padilla, O Que é Missão Integral?, 14; M. Raiter, "Enviados para esse propósito: 'missão', 'missiologia' e a busca por um significado", in Missão Integral Transformadora, ed. M. W. Kohl e A. C. Barro (Londrina, PR: Descoberta, s.d.), 71; Johannes Verkuyl, Contemporary Missiology: an introduction (Grand Rapids, MI: Wm. B. Eerdmans, 1978), 3-4.

23 Guimarães, "Compreendendo 
El plantío de iglesias, que se utiliza en nuestros días, trata uno de los métodos utilizados por los seguidores de Jesucristo para cumplir la gran comisión, que a saber es, hacer discípulos de todas las naciones (Mt 28:19. 20). Junto con la tarea ordenada por Jesús a su iglesia, los discípulos se fortalecieron con las promesas de los cuatro "todo" relacionado con la misión: ${ }^{24}$

1. Toda autoridad tiene el que los envía.

2. Todas las naciones, pueblos y grupos étnicos de la tierra deben recibir el mensaje de Jesús.

3. Todas las cosas que fueron ordenadas por Jesús

e Definindo os Termos Relacionados à Missão"; Zwetsch, "Missão - téstemunho do evangelho no horizonte do reino de Deus", 214-215; Jorge Henrique Barro, ed., O pastor Urbano: dez desafios práticos para uma nova descoberta, $2^{\mathrm{a}}$ ed. (Londrina, PR: Descoberta, 2008), 15-20; Verkuyl, Contemporary Missiology, 3-5.

24 Abdala, Guia de Plantío de Igrejas, 11; George W. Peters, A Theology of Church Growth (Grand Rapids, MI: Zondervan, 1981), 10-20; George W. Peters, Teologia Bíblica das Missões, $4^{\mathrm{a}}$ ed. (Rio de Janeiro: CPAD, 2004), 16. se les debe enseñar.

4. Todos los días, la autoridad de Jesús continuaría con ellos y con todos los demás seguidores hasta el fin de la historia de este mundo. ${ }^{25}$

Schantz dice que la respuesta de la iglesia a la gran comisión de Jesús consiste en tres "todo" y "una" posibilidad. Pablo, y por implicación la Iglesia cristiana, respondió a los cuatro "todo" con una declaración registrada en 1 Co 9:19-22:26:

1. Me hice de todo. Los versículos 20-22 muestran la capacidad de adaptación del seguidor de Jesús a las nuevas situaciones. Se necesita la sabiduría y la comprensión de la naturaleza humana, para

25 Borge Schantz, A Path Straight to the Hedges: Evangelism in the Developing Areas (Nampa, ID: Pacific Press, 2000), 19; Bosch, Missão Transformadora, 27-28; Justo L. González y Carlos Cardoza Orlandi, História do Movimento Missionário (São Paulo, SP: Hagnos, 2010), 23-24.

26 Schantz, A Path Straight to the Hedges, 21. 
distinguir entre los absolutos de la Biblia y lo que es opcional. ${ }^{27}$

2. Para con todos. Este "todos" de Corintios corresponde con el término "todas las naciones" de la gran comisión de Mateo.

3. Para que de todos los modos. El tercer "todos" dice que los métodos misioneros no deben ser considerados inflexibles. Si bien los principios y mandamientos bíblicos son inmutables, los métodos pueden ser contextualizados y adaptados.

4. Salvar a algunos - no todos serán salvos. Así Pablo, al hablar de los resultados de la misión, cambia la palabra, de "todos" a "algunos". 28

Como el campo objetivo del esfuerzo misionero, usa la pa-

27 Elena G. de White también tiene ese pensamiento. Ver, por ejemplo: Elena G. de White, Obreros evangélicos (Doral, FL: Publicaciones Interamericanas, 2003), 123-125.

28 Schantz, 21. labra naciones - ethne - Mateo 28:19, que se refiere a todos los grupos nacionales, culturales, religiosos y raciales. A medida en que la gran comisión requiere que se hagan discípulos en cada grupo de personas, una iglesia por país, ciudad o barrio no es suficiente para alcanzar a todos los grupos sociales. Así, la iglesia debe establecer nuevas iglesias entre nuevos grupos de personas hasta la venida de Jesús. ${ }^{29}$

Cuatro grupos culturales fueron mencionados por Jesús a sus discípulos en su ascensión. Hechos 1:8 menciona cuatro áreas geográficas que componen la gran comisión:

1. Jerusalén. Capital, incluyendo las mediaciones. Lugar donde ellos estaban.

2. Judea. Provincia cuya ca-

29 Russel Burril, Discípulos Modernos (Tatuí, SP: Casa Publicadora Brasileira, 2006), 60; Peters, A Theology of Church Growth, 20-30; Peters, Teologia Bíblica das Missões, 12-24; Jim Petersen, Evangelism as a Life Style (Colorado Springs, CO: NavPress, 1980), 36-39. 
pital era Jerusalén. Lugares cercanos.

3. Samaria. Provincia cercana de Judea. Era una transición entre el pueblo de Dios y los gentiles, porque ellos eran sincretistas en materia de religión. La gente en transición ofrece un gran entrenamiento para los cristianos que irán a los confines de la tierra. Lugares distantes, pero con similitudes.

4. Confines de la tierra. Son personas de diferentes idiomas, diferentes colores, diferentes religiones. ${ }^{30}$

Así, el movimiento misionero cristiano que se inició en el aposento alto, con 120 seguidores de Cristo (Hch 1:15), terminó su informe numérico mencionando que miles de judíos creían (Hch 21:20). Lucas, como el autor del libro de los

30 Burril, Discípulos Modernos, 3031; Peters, A Theology of Church Growth, 16; Peters, Teologia Bíblica das Missões, 18-20; Petersen, Evangelism as a Life Style, $22-25$.
Hechos, parece que igualó las prácticas de suma y multiplicación en su descripción de crecimiento. Dos veces la palabra adición aparece, una inmediatamente después de Pentecostés en Hechos 2:41, 47, y otra es mencionada en Lucas 5:14; 11:24. Esto muestra que aparentemente hay un concepto de expansión de la iglesia local en número y tamaño. ${ }^{31}$

Sin embargo, el término multiplicación aparece en Hechos $6: 1,7 ; 9: 31 ; 12: 24$. En estos textos se presenta que los discípulos y las iglesias crecían y se multiplicaban. Tres iglesias se destacan: Jerusalén (Hch 1-7; 6:7), Antioquía en Siria (Hch 11:19-30; 13:1-3) y Roma (Hch 28:15; Rm 1:8-13). En la práctica apostólica, la adición (ampliación) precede a la multiplicación (extensión). Como no es prudente multiplicar dejando numerosos grupos con un mal funcionamiento, el entrenamiento es absolutamente esen-

31 Peters, A Theology of Church Growth, 193. 
cial, para lograr el ideal bíblico de crecimiento de la iglesia. ${ }^{32}$

\section{Pablo y el plantío de iglesias}

El apóstol Pablo usó las palabras edificar y plantar cuando se refirió al trabajo en Corinto (1 Co 3:5-11). En Hechos 9:31, Pablo iguala claramente la conversión de las personas con la organización de las iglesias. Su objetivo principal era establecer iglesias en cada ciudad importante del Asia Menor, siempre teniendo en cuenta el lugar donde Cristo era desconocido. ${ }^{33}$

En su dedicación evangelizadora, Pablo planeó el desarrollo de nuevas congregaciones en

32 Ibíd.; Thom S. Rainer, The Book of Church Growth (Nashville, TN: B\&H, 1998), 20-30; Amberson, Birth of Church, 12-17; Chaney, Church Planting and the End of Twentieth Century, 21-22; Dudley and Gruesbeck, Plant a Church, Reap a Harvest, 36-38; Faircloth, Church Planting for Reprodution, 15-17.

33 Emílio Abdala, "Como Plantar Igrejas", Revista do Ancião, junio 2011; Abdala, Guia de Plantio de Igrejas, 13; Allen, Missionary Methods, St. Paul's or Ours?, 23-25. "nuevas fronteras" (2 Co 10:1316). En todo esto, por supuesto, obedeció a la dirección del Espíritu Santo (Hch 16:7-13). ${ }^{34}$

Pablo estableció muchas nuevas iglesias: Tesalónica (Hch 17:1-4), Berea (Hch 17:10-12), Corinto (Hch 17:1-17), Éfeso (Hch 18:18-21; 19:1-41), son ejemplos de iglesias plantadas por él.

En Hechos 16 ocurrió el establecimiento de la iglesia en $\mathrm{Fi}$ lipo (Hch 16). Pablo no tuvo el privilegio de permanecer mucho tiempo en Filipo, porque tuvo que salir de inmediato. Antes de eso, él organizó y alentó a la iglesia. No llevó líderes del exterior, sino que confió el trabajo a los líderes nativos. ${ }^{35}$

34 Hesselgrave, Planting Churches, 28-30; Gary L. McIntosh, Biblical Church Growth: How You Can Work with God to Build a Faithful Church (Grand Rapids, MI: Baker, 2003), 10-14; Stephan Neill, A History of Christian Missions (Middlesex, UK: Penguin, 1986), 23-25.

35 Allen, Missionary Methods, St. Paul's or Ours?, 45-47; Hesselgrave, Planting Churches, 30-32; Peters, A Theology of Church Growth, 28-30. 
Por lo tanto, aquí tenemos un modelo a seguir, es decir, capacitar y confiar el liderazgo a los miembros locales para seguir trabajando. Las cartas paulinas son evidencia de la constante preocupación por las iglesias fundadas y el proceso de discipulado de los nuevos miembros. ${ }^{36}$

\section{La iglesia de Antioquía}

Probablemente, desde su trabajo inicial en la iglesia de Antioquía en Siria junto con Bernabé, Pablo había asimilado un modelo digno de ser repetido en todo el mundo: una iglesia comprometida a plantar otras iglesias.

El libro de los Hechos ha sido llamado "la historia de las tres ciudades". Lucas comienza su relato con los grandes milagros en Jerusalén, y luego cambia su enfoque hacia una ciudad casi desconocida llamada Antioquía.

Al final del libro de los $\mathrm{He}$ chos, el centro del testimonio

36 Chaney, Church Planting and the End of Twentieth Century, 21-22; Hesselgrave, Planting Churches, 28-30. parece ser la ciudad de Roma, donde Pablo escribe algunas de sus epístolas. La iglesia de Antioquía podría no tener la importancia profética de las siete iglesias mencionadas en Apocalipsis, pero de ella se puede aprender mucho del crecimiento, la vitalidad y relevancia para nuestras iglesias hoy en día. ${ }^{37}$

\section{Marco conceptual del plantío de iglesias}

Rufus Anderson expone el plantío de iglesias como un objetivo basado en cuatro áreas:

1. La conversión de los perdidos.

2. Su organización en igle-

37 Ken Parker, Seven Characteristics of a Growing Church (Pasadena, CA: Church Growth, 1979), 61; Artur G. Patzia, The Emergence of the Church: Context, Growth, Leadership \& Worship (Downers Grove, IL: InterVarsity, 2001), 98; Michael Green, Evangelização na Igreja Primitiva (São Paulo: Vida Nova, 1970), 38; Michael Green, Evangelism Now and Then (Leicester, UK: InterVarsity, 1979), 34; Rodney Stark, The Rise of Christianity: a sociologist reconsiders history (Princeton, NJ: Princeton University Press, 1996), 158-161. 
sias locales.

3. Promoción y formación de líderes en cada comunidad.

4. Fomento de la independencia espiritual y de organización en cada comunidad. $^{38}$

Entre tantos puntos que definen el término plantío de iglesias, en la Biblia y en la teología, Ronaldo Lidório expresa los principios enunciados de modo coherente en tres puntos:

1. El plantío de iglesias no debe ser definido en términos de entrenamiento y habilidad, sino por el poder y el deseo de Dios para salvar vidas.

2. El plantío de iglesias no debe ser definido en términos de resultados humanos, sino por la fidelidad a la Sagrada Escritura.

3. El plantío de iglesias no debe ser una acción defi-

38 Rufus Anderson, Foreign Missions: Their Relations and Claims (New York: Scribners, 1869), 5 . nida por el conocimiento del Evangelio, sino por su proclamación. ${ }^{39}$

Lidório resume: "No importa lo que haga un sembrador de iglesias, él debe proclamar el evangelio. El trabajo social, el ministerio holístico y comprensión cultural, nunca remplazarán la comunicación clara del evangelio o la presencia de la iglesia". 40

El contenido del evangelio expuesto en cualquier ministerio de plantío de iglesias debe incluir:

1. Dios como el Creador y Soberano (Ef 1:3-6).

2. La Biblia como la Palabra inspirada de Dios (2 Ti 3:16).

39 Ronaldo Lidório, "O que define a plantação de igrejas, teológica e biblicamente?", Instituto Antropos - Pesquisa Social e Missiologia Aplicada, 19 de abril, 2011, http://instituto.antropos.com.br/v3/ index.php?option $=$ com_content $\& v i e w=$ article\&id=613:o-que-define-a-plantacao-de-igrejas-teologica-e-biblicamente- $\&$ catid $=42$ : consultoria- onlineem-plantío-de-igrejas \&Itemid $=58$ (consultado: 25 de julio, 2012).

40 Lidório,"RonaldoeRossanaLidório - Teologia bíblica da contextualização". 
3. El pecado como fuente de separación entre el hombre y Dios (Ef 2:5).

4. La gran controversia entre Cristo y Satanás (Ap 12).

5. Jesús, su cruz, la resurrección y el ministerio en el santuario celestial, como centro histórico de Dios y centro para la redención del hombre (Heb 1:1-4);

6. El Espíritu Santo como el cumplimiento de la promesa y responsable de guiar a la iglesia hasta el último día (Jn 14:16).

227 7. Los mandamientos de Dios como permanentes y el deber de todo cristiano (Ec 12:13-14). ${ }^{41}$

\section{Elena G. de White y el plantío de iglesias}

En general, se encuentran en los escritos de Ellen White los siguientes consejos sobre el establecimiento de nuevas iglesias.

41 Associação Geral da Igreja Adventista do Sétimo Dia, Manual da Igreja Adventista do Sétimo Dia (Tatuí, SP: Casa Publicadora Brasileira, 2011), 163-173.

\section{Para la conversión de los perdidos}

Nuestra obra misionera debe ser más agresiva hoy que en el pasado. Debemos añadir nuevos territorios; la norma de la verdad debe ser establecida en nuevos lugares; hay que fundar iglesias; hay que hacer todo lo posible para cumplir este cometido: "Por tanto, id, y haced discípulos a todas las naciones". ${ }^{42}$

\section{Para alcanzar a todos las regiones}

"En toda ciudad, donde se proclama la verdad, deben fundarse iglesias. En algunas ciudades grandes debe haber iglesias en varias partes de la ciudad". ${ }^{4}$

\section{Para desarrollar los dones espirituales}

Sobre todos los que creen, Dios ha colocado la responsabi-

42 Elena G. de White, Cada día con Dios (Doral, FL: Publicaciones Interamericanas, 2003), 240.

43 Elena G. de White, Obreros evangélicos (Doral, FL: Publicaciones Interamericanas, 2003), 410. 
lidad de levantar iglesias con el expreso propósito de educar a hombres y mujeres para que utilicen, en beneficio del mundo, las aptitudes que les ha concedido y empleen para su gloria los medios que les ha confiado. ${ }^{44}$

\section{Para que ocurra la inde- pendencia y liderazgo espiritual}

A medida que se establezcan iglesias, debe hacerles entender que aun de entre ellas ha de tomarse hombres para que lleven la verdad a otros y hagan surgir nuevas iglesias; por lo tanto, todos deben trabajar y cultivar hasta el máximo los talentos que Dios les ha dado, educando sus mentes para empeñarse en el servicio de su Maestro. ${ }^{45}$

Entonces, durante su ministerio, Elena G. de White instó a los adventistas para:

1. Desarrollar una estrategia para establecer iglesias

44 Ibíd., 419.

45 Elena G. de White, Servicio cristiano (Doral, FL: Publicaciones Interamericanas, 2003), 77. que ponga al adventismo en cada ciudad y villa.

2. En lugar de utilizar las mismas personas para abrir varias iglesias, ella quería que las nuevas iglesias provean nuevas personas para el proceso de plantío de iglesias.

3. Ella consideraba el asunto como la función primaria de la Iglesia adventista. Todas las otras actividades deberían ajustarse a un programa agresivo para alcanzar todas las áreas descuidadas. ${ }^{46}$

\section{Propósito}

Como el trabajo tiene un carácter retrospectivo, se descri-

46 Elena G. de White, El ministerio médico (Doral, FL: Publicaciones Interamericanas, 2003), 309-320; Elena G. de White, Mensajes selectos (Doral, FL: Publicaciones Interamericanas, 2003), 1:112; White, Obreros evangélicos, 345; White, Testimonios para la iglesia, 8:244; Elena G. de White, El evangelismo (Doral, FL: Publicaciones Interamericanas, 2003), 19-21; 47; White, Testimonios para la iglesia, 2:340; White, Servicio cristiano, 71; White, Testimonios para la iglesia, 6:24. 
birán los principios fundamentales del crecimiento de iglesia encontrados en el estudio.

\section{Metodología}

La investigación es descriptiva, cuantitativa y retrospectiva. Es descriptiva porque describe la información obtenida. Es cuantitativa porque los datos son expuestos numéricamente. Es retrospectiva porque se exponen hechos ocurridos en el pasado.

Las fuentes de información 24 que se utilizaron fueron básicamente secundarias. Las fuentes primarias son entrevistas a algunos miembros de la Iglesia adventista de Eunapolis que vivieron en esos años.

El diseño es no experimental porque no se manipula ninguna variable y de corte longitudinal porque el estudio abarca los años1980 a 2010.

La población y la muestra de este estudio de investigación fue tomada de feligreses ubicados en la zona metropolitana de la ciudad de Eunapolis, entre los años 1980 y 2010.

Los datos fueron obtenidos a partir de entrevistas con fundadores, archivos de la Secretaría de la Iglesia adventista central de Eunapolis, informes estadísticos de los archivos de la Secretaría de la Asociación Bahía Sur de la Iglesia Adventista del Séptimo Día, informes estadísticos anuales de la Asociación General de la Iglesia Adventista del Séptimo Día y los informes estadísticos de los archivos del Instituto Brasileño de Geografía y Estadística.

\section{Resultados de la estrategia de plantío de iglesias adventistas en la zona metropolitana de Eunapolis}

\section{Breve recorrido histórico del adventismo en Eunapolis}

La historia del adventismo en Eunapolis está marcada por una dinámica actuación de los miembros voluntarios, ya que al principio la distancia de la 
ciudad a la sede del distrito misionero era muy distante, y los pastores aparecían en intervalos de tiempo muy grandes (de un año a otro, e incluso más). ${ }^{47}$

El adventismo en Eunapolis comenzó con Narciso Sacramento y su familia, en el año 1956. Habían aceptado a Jesús en Nova Conquista (actual Candido Sales), interior de Bahía, entre los años 1953 y 1954. A partir de 1960, llegó a Eunapolis la familia Moraes y Antonio Santos.

Entonces decidieron construir un pequeño templo en un lugar distante y aislado, lo que hoy es el barrio de Colonia. ${ }^{48}$

En el año 1966 se trasladó a Eunapolis un converso de la Voz de Profecía, Ebrahim Peixoto. En ese mismo año, Ebrahim consiguió el salón de la iglesia presbiteriana que se ubicaba en

47 Moisés Vieira Santos, "Entrevista Sobre a História da Igreja Adventista em Eunápolis", entrevista por Heraldo Vander Lopes, 2 de mayo, 1996.

48 Sacramento, "Entrevista Sobre a História da Igreja Adventista em Eunápolis". el barrio de Pequi, para que en los sábados celebren sus reuniones religiosas. ${ }^{49}$

En 1968 se mudaron a una pequeña casa ubicada en el centro de la ciudad, pero la ubicación resultó ser incorrecta. Volvieron, pues, para el barrio de Pequi, y se congregaron en la residencia de Ebrahim Peixoto. ${ }^{50}$

Ese mismo año, Moisés Vieira donó una casa en la calle del Pescado (ahora Paulino Mendes). Permanecieron allí, hasta que Clarita Messias donó un terreno en la calle Joaquim Alves Pereira, N. ${ }^{\circ} 247$, donde se construyó un pequeño templo. Se mudaron a este lugar en $1971 .^{51}$

Simultáneamente, en el año 1970, la familia Viana Braga se mudó para Guaratinga, fundando una iglesia allí. Lo mismo

49 Ebrahim Peixoto, "Entrevista Sobre a História da Igreja Adventista em Eunápolis", entrevista por Heraldo Vander Lopes, 7 de mayo, 1996.

50 Ibíd.

51 Santos, "EntrevistaSobreaHistória da Igreja Adventista em Eunápolis”. 
ocurrió en Itabela, con la familia Silva fundando la iglesia allí también, en 1970.52 Sin embargo, esas iglesias no se han establecido por iniciativa de Eunapolis, sino porque las familias se han trasladado a otro lugar.

El año 1973 fue marcado por una serie de reuniones públicas que el pastor Luis Santana realizó, donde se bautizaron 33 personas. En el año 1974, la iglesia se convirtió en la sede del distrito misionero, y el 22 de junio de 1974, el grupo pasó a ser iglesia 26 organizada, con sesenta y cinco miembros. ${ }^{53}$

Con el desarrollo de la industria de extracción y procesamiento de la madera en la década de los 70', hubo una gran migración de personas para la región, principalmente de capixabas. Esto trajo una nueva mentalidad para la región. Con

52 Jair Passos, "Entrevista Sobre a História da Igreja Adventista em Eunápolis".

53 Secretaria da Igreja Adventista Central de Eunápolis, "Ata de Organização da Igreja Adventista Central de Eunápolis", 22 de junio, 1974. su espíritu expansionista, aliado a la prosperidad financiera que poseían, Jair Pasos y Emilio Damm, lideraron la fase de plantación de nuevas iglesias. ${ }^{54}$

\section{En 1978 compraron un gran} terreno y contrataron a un maestro laico de la iglesia, conocido como Elías Freitas..$^{55}$ Bautizaron trescientos catorce personas. Entonces, construyeron un gran templo y fundaron la Iglesia adventista del barrio de Pequi, conocida por ABBI (Asociación Brasileña de Bienestar Integral), el nombre de las reuniones públicas para atraer a la gente. Hoy en día, $A B B I$ sigue siendo la iglesia adventista más grande en el extremo sur de Bahía, con 695 miembros. ${ }^{56}$

En ABBI, y en las nuevas iglesias que fueran fundadas, ocurrieron los cuatro principios bí-

54 Passos, "Entrevista Sobre a História da Igreja Adventista em Eunápolis".

55 Elias Freitas é pseudónimo.

56 Secretaria da Associação Bahia Sul (ABS), Distritos e Igrejas em 31/12/2010. 
blicos, según Rufus Anderson, ${ }^{57}$ para el establecimiento de nuevas iglesias:

1. Conversión de los perdidos, con reuniones públicas seguidas por bautismos de quienes aceptaron el mensaje adventista.

2. Organización de iglesias locales, con construcción de templos.

3. Promoción y formación de líderes en cada comunidad, con la organización de la iglesia y establecimiento de líderes locales.

4. Fomento de la independencia espiritual y de organización en cada comunidad, con una actitud en las iglesias fundadas para establecer nuevas iglesias, a través de un trabajo conjunto con la Iglesia central de Eunapolis.

En 1979, fueron al barrio de Gusmão e hicieron el mismo tra-

57 Anderson, Foreign Missions: Their Relations and Claims, 5; Beaver, "The Legacy of Rufus Anderson". bajo, con los mismos principios. Como resultado, noventa y siete personas fueron bautizadas, y una iglesia más fue construida y organizada. En el año 1980 tocó el turno al barrio de Juca Rosa, y otra iglesia fue construida y también organizada. Lo mismo ocurrió en la villa de São João do Sul..$^{58}$

La década de los 70 terminó con siete iglesias y quinientos sesenta y nueve miembros. El movimiento de plantío de iglesias comenzó en 1978, con la fundación de la iglesia de ABBI. El plantío de iglesias, iniciado con $\mathrm{ABBI}$, motivó la fundación de más iglesias. Desde entonces, fue la estrategia adoptada para el crecimiento del adventismo en la región. Véase la Tabla 1.

A partir de 1980, se convierte en la estrategia adoptada para el crecimiento del adventismo en la región. Esa década terminó con diecisiete iglesias y mil tres-

58 Bráulio Thomé Júnior, "Entrevista Sobre a História da Igreja Adventista em Eunápolis", entrevista por Heraldo Vander Lopes, 1 de mayo, 1996. 
cientos ochenta y ocho miembros. El plantío de iglesias demostró ser una estrategia eficaz para la región.

En la década de los 90, el plantío de iglesias aumentó. Veinte nuevas iglesias fueron establecidas. El adventismo llegó a todos los municipios de la zona metropolitana de Eunapolis. En 1989, se reanudó el establecimiento de nuevas iglesias, y de allí en adelante todos los años, por lo menos una nueva iglesia era plantada e implementada.

La década de los noventa terminó con treinta y siete iglesias y dos mil quinientos cincuenta miembros. El plantío de iglesias continuó siendo la estrategia utilizada en la región. Véase la Tabla 2.

A principios del nuevo siglo, el plantío de iglesias permaneció siendo fuerte. Treinta y nueve congregaciones más se añadieron. El año 2006 hubo un movimiento evangelístico y plantación de iglesias en la región; todas las iglesias ya establecidas simultáneamente participaron en el movimiento. Como resultado, más de dos mil personas fueron bautizadas y cuatro nuevas iglesias fueron fundadas: Fruto da Terra, Monte Azul, Santiago y Alto da Boa Vista. Véase la Tabla 3.

Con el fin de ver mejor lo que ocurrió, el crecimiento se expresará en porcentaje. La fórmula matemática para calcular el incremento es:

$$
\mathrm{IP}=\left(\frac{\mathrm{Pi}-\mathrm{Pf}}{\mathrm{Pi}}\right) \times 100
$$

Donde,

IP: Incremento Poblacional (expresado en porcentaje - \%);

Pi: Población inicial;

Pf : Población final. ${ }^{59}$

Esta es la misma fórmula para calcular tanto el crecimiento anual como lo decenal. ${ }^{60}$

59 wikiHow, "Como Calcular Taxa de Crescimento - wikiHow”, http://pt.wikihow. com/Calcular-Taxa-de-Crescimento (consultado: 24 de septiembre, 2012).

60 La Tasa de Crecimiento Anual 
Tabla 1. Año del plantío de la iglesia, cantidad de iglesias y de feligreses, crecimiento en \% (1981-1990) ${ }^{61}$.

\begin{tabular}{|c|c|c|c|c|c|c|}
\hline Año & $\begin{array}{c}\text { Cantidad } \\
\text { de iglesias } \\
\text { plantadas en } \\
\text { el año }\end{array}$ & $\begin{array}{c}\text { Iglesias } \\
\text { plantadas }\end{array}$ & $\begin{array}{c}\text { Cantidad } \\
\text { de iglesias } \\
\text { al final del } \\
\text { año }\end{array}$ & $\begin{array}{c}\text { Crecimiento } \\
\text { de iglesias } \\
\text { en \% }\end{array}$ & $\begin{array}{c}\text { Total de } \\
\text { feligreses en } \\
\text { el año }\end{array}$ & $\begin{array}{c}\text { Crecimiento } \\
\text { de feligreses } \\
\text { en \% }\end{array}$ \\
\hline 1981 & 1 & Urbis 1 & 8 & $14,29 \%$ & 694 & $23,71 \%$ \\
\hline 1982 & 1 & Itapebi & 9 & $12,50 \%$ & 757 & $9,08 \%$ \\
\hline 1983 & 1 & $\begin{array}{c}\text { Barrolân- } \\
\text { dia }\end{array}$ & 10 & $11,11 \%$ & 831 & $9,78 \%$ \\
\hline 1984 & 1 & $\begin{array}{c}\text { Vale } \\
\text { Verde }\end{array}$ & 11 & $10,00 \%$ & 895 & $7,70 \%$ \\
\hline 1985 & 3 & $\begin{array}{c}\text { Km } 46, \\
\text { Porto } \\
\text { Seguro, } \\
\text { Belmonte }\end{array}$ & 14 & $27,27 \%$ & 1.085 & $21,23 \%$ \\
\hline 1986 & - & - & 14 & $0,00 \%$ & 1.130 & $4,15 \%$ \\
\hline 1987 & - & - & 14 & $0,00 \%$ & 1.177 & $4,16 \%$ \\
\hline 1988 & - & - & 14 & $0,00 \%$ & 1.224 & $3,99 \%$ \\
\hline 1989 & 1 & $\begin{array}{c}\text { Mundo } \\
\text { Novo }\end{array}$ & 15 & $7,14 \%$ & 1.299 & $6,13 \%$ \\
\hline 1990 & 2 & $\begin{array}{c}\text { Cambo- } \\
\text { lo, Vera } \\
\text { Cruz }\end{array}$ & 17 & $13,33 \%$ & 1.388 & $6,85 \%$ \\
\hline
\end{tabular}

(TCA) y la Tasa de Crecimiento Decenal (TCD), es una medida estandardizada que compara el crecimiento de la Iglesia de un año a otro o de una década a otra. Esta puede ser positiva o negativa. Bob Waymire y C. Peter Wagner, Manual de análisis de iglecrecimiento, 3a ed. (Santa Clara, CA: Global Church Growth, 1983), 15-16.

61 Secretaría de la Iglesia Adventista Central de Eunapolis, Secretaría de la Asociación Bahía Sur (ABS) de la IASD. 
Tabla 2. Año del plantío de la iglesia, cantidad de iglesias y de feligreses, crecimiento en \% $(1991-2000)^{62}$.

\begin{tabular}{|c|c|c|c|c|c|c|}
\hline Año & $\begin{array}{c}\text { Cantidad } \\
\text { de iglesias } \\
\text { plantadas en } \\
\text { el año }\end{array}$ & $\begin{array}{l}\text { Iglesias plan- } \\
\text { tadas }\end{array}$ & $\begin{array}{c}\text { Cantidad } \\
\text { de iglesias } \\
\text { al final del } \\
\text { año }\end{array}$ & $\begin{array}{c}\text { Cre- } \\
\text { cimiento } \\
\text { de iglesias } \\
\text { en } \%\end{array}$ & $\begin{array}{l}\text { Total de } \\
\text { feligreses } \\
\text { en el año }\end{array}$ & $\begin{array}{l}\text { Creci- } \\
\text { miento } \\
\text { de fe- } \\
\text { ligreses } \\
\text { en } \%\end{array}$ \\
\hline 1991 & 2 & $\begin{array}{c}\text { Minas Gerais, } \\
\text { Coroa Vermelha }\end{array}$ & 19 & $11,76 \%$ & 1.479 & $6,56 \%$ \\
\hline 1992 & 1 & Moisés Reis & 20 & $5,26 \%$ & 1.583 & $7,03 \%$ \\
\hline 1993 & 1 & Baianão & 21 & $5,00 \%$ & 1.697 & $7,20 \%$ \\
\hline 1994 & 2 & $\mathrm{Km} 12$ e Urbis 3 & 23 & $9,52 \%$ & 1.750 & $3,12 \%$ \\
\hline 1995 & 3 & $\begin{array}{c}\text { Imbiruçu de } \\
\text { Dentro, Projeto } \\
\text { Maravilha,Santa } \\
\text { Lúcia }\end{array}$ & 26 & $13,04 \%$ & 1.836 & $4,91 \%$ \\
\hline 1996 & 4 & $\begin{array}{c}\text { Alecrim, Agro- } \\
\text { vila, Itagimirim, } \\
\text { Santa Cruz Ca- } \\
\text { bralia }\end{array}$ & 30 & $15,38 \%$ & 1.983 & $8,01 \%$ \\
\hline 1997 & 3 & $\begin{array}{c}\text { Parque } \\
\text { Ecológico,Bairro } \\
\text { da Alegria, } \\
\text { Ponto Central }\end{array}$ & 33 & $10,00 \%$ & 2.198 & $10,84 \%$ \\
\hline 1998 & 1 & Paraguai & 34 & $3,03 \%$ & 2.390 & $8,74 \%$ \\
\hline 1999 & 1 & $\begin{array}{c}\text { Mercado do } \\
\text { Povo }\end{array}$ & 35 & $2,94 \%$ & 2.471 & $3,39 \%$ \\
\hline 2000 & 2 & $\begin{array}{c}\text { Pindorama, } \\
\text { Monte Pascoal }\end{array}$ & 37 & $5,71 \%$ & 2.550 & $3,20 \%$ \\
\hline
\end{tabular}

62 Secretaría de la Iglesia Adventista Central de Eunapolis, Secretaría de la Asociación Bahía Sur (ABS) de la IASD. 
Tabla 3. Año del plantío de la iglesia, cantidad de iglesias y de feligreses, crecimiento en $\%(2001-2011)^{63}$.

\begin{tabular}{|c|c|c|c|c|c|c|}
\hline Año & $\begin{array}{l}\text { Cantidad } \\
\text { de iglesias } \\
\text { plantadas } \\
\text { en el año }\end{array}$ & Iglesias plantadas & $\begin{array}{c}\text { Cantidad } \\
\text { de iglesias } \\
\text { al final del } \\
\text { año }\end{array}$ & $\begin{array}{l}\text { Cre- } \\
\text { cimiento } \\
\text { de igle- } \\
\text { sias en \% }\end{array}$ & $\begin{array}{c}\text { Total de } \\
\text { feligreses } \\
\text { en el año }\end{array}$ & $\begin{array}{l}\text { Creci- } \\
\text { miento de } \\
\text { feligreses } \\
\text { en } \%\end{array}$ \\
\hline 2001 & 5 & $\begin{array}{c}\text { Mirante Caravelas, Ouro } \\
\text { Verde, Itapoan,Bairro da } \\
\text { Tânia, Camurugi }\end{array}$ & 42 & $13,51 \%$ & 2.614 & $2,51 \%$ \\
\hline 2002 & 1 & Sapucaeira & 43 & $2,38 \%$ & 2.690 & $2,91 \%$ \\
\hline 2003 & 2 & Vila Jardim, Rosa Neto & 45 & $4,65 \%$ & 2.746 & $2,08 \%$ \\
\hline 2004 & 2 & Arraial d'Ajuda, Trancoso & 47 & $4,44 \%$ & 3.064 & $11,58 \%$ \\
\hline 2005 & 2 & $\begin{array}{c}\text { Campo Verde, Dinah } \\
\text { Borges }\end{array}$ & 49 & $4,26 \%$ & 3.200 & $4,44 \%$ \\
\hline 2006 & 4 & $\begin{array}{c}\text { Fruto da Terra, Monte } \\
\text { Azul, Santiago,Alto da } \\
\text { Boa Vista } \\
\end{array}$ & 53 & $8,16 \%$ & 5.339 & $66,84 \%$ \\
\hline 2007 & 4 & $\begin{array}{l}\text { Casas Novas, Vila Vitória, } \\
\text { Bairro Novo, Esperança }\end{array}$ & 57 & $7,55 \%$ & 5.653 & $5,88 \%$ \\
\hline 2008 & 5 & $\begin{array}{l}\text { Estela Reis, Vida Total, } \\
\text { Bandeirantes,Novo Hori- } \\
\text { zonte, Roça do Povo }\end{array}$ & 62 & $8,77 \%$ & 6.071 & $7,39 \%$ \\
\hline 2009 & 4 & $\begin{array}{l}\text { Nilo Fraga, Colônia, } \\
\text { Vila Olímpica, Itaporanga }\end{array}$ & 66 & $6,45 \%$ & 6.302 & $3,80 \%$ \\
\hline 2010 & 5 & $\begin{array}{l}\text { Recanto das Árvores, } \\
\text { Colonial,Quinto Cen- } \\
\text { tenário, Carajá, Vila Beata }\end{array}$ & 71 & $7,58 \%$ & 6.705 & $6,39 \%$ \\
\hline 2011 & 5 & $\begin{array}{c}\text { Cajueiro, Fontana, Cen- } \\
\text { tauro, Buranhém, Parque } \\
\text { Renovação }\end{array}$ & 76 & $7,04 \%$ & 7.081 & $5,61 \%$ \\
\hline
\end{tabular}

63 Secretaría de la Iglesia Adventista Central de Eunapolis, Secretaría de la Asociación Bahía Sur (ABS) de la IASD. 
Entre 1970 y 1980, el número de iglesias adventistas en Brasil creció de 2.168 a 3.055 iglesias, es decir, hubo un incremento del 40,91\%. En ese momento, el número de iglesias adventistas en la zona metropolitana de Eunapolis, Bahía (Brasil), aumentó de 3 a 7 , un incremento de $133,33 \%$. Los adventistas en Brasil aumentaron de 161.187 miembros a 280.664, es decir, hubo un aumento de $74,12 \%$. En esa misma época, los adventistas en el área metropolitana de Eunapolis crecieron de 36 a 561 miembros,

32 es decir, hubo un aumento de $1.458,33 \%$. Por lo tanto, la tasa de crecimiento en el número de iglesias y miembros en el área metropolitana de Eunapolis fue mayor que la tasa de crecimiento de la población en Brasil y de la Iglesia Adventista en el país durante el mismo período.

En la década de los 80 la estrategia de plantío de iglesias fue adoptada definitivamente por los feligreses adventistas en el área metropolitana de Eunapolis, ya que los resultados alcanzados eran visibles y la estrategia coherente con sus creencias y tradiciones, ya que la ejecución era simple y eficiente.

Entre 1980 y 1990, la población de Brasil pasó de 121.150.573 a 146.917.459 habitantes, creciendo 21,26\%. En este mismo período, la población de Eunapolis pasó de 90.795 a 111.741 habitantes, creciendo 23,06\%. El número de iglesias adventistas en Brasil creció, en este tiempo de 3.055 a 5.334 iglesias, es decir, hubo un incremento del $74,59 \%$.

En ese momento, el número de iglesias adventistas en la zona metropolitana de Eunapolis, Bahía, Brasil, aumentó de 7 a 17, incrementándose en 142,85\%. Finalmente los adventistas en Brasil aumentaron de 280.664 miembros a 541.186, es decir, aumentaron en un $92,82 \%$ y los adventistas, en el área metropolitana de Eunapolis, crecieron de 561 a 1.388 miembros, es decir, hubo un aumento de $147,41 \%$. Veáse la Tabla 4. 
Por lo tanto, la tasa de crecimiento de los adventistas en Eunapolis continuó mayor que la tasa de crecimiento de la población en Brasil y de la Iglesia adventista en el país, durante el mismo período.
En la década de los noventa, la estrategia de plantío de iglesias en el área metropolitana de Eunapolis se mantuvo estable, siempre superando el crecimiento en Brasil.

\section{Tabla 4. Crecimiento comparativo de la IASD en Brasil y en la zona me- tropolitana de Eunapolis (1980-1990) ${ }^{64}$}

\begin{tabular}{|c|c|c|c|c|}
\hline LUGAR & AÑOS & 1980 & 1990 & $\%$ \\
\hline \multirow{5}{*}{ BRASIL } & Habitantes & 121.150 .573 & 146.917 .459 & $21,26 \%$ \\
& Iglesias & 3.055 & 5.334 & $74,59 \%$ \\
& Miembros & 280.664 & 541.186 & $92,82 \%$ \\
& Miembros/habitantes & $1 / 424$ & $1 / 271$ & - \\
\hline \multirow{3}{*}{ EUNAPOLIS } & & 90.795 & 111.741 & $23,06 \%$ \\
& Habitantes & 7 & 17 & $142,85 \%$ \\
& Iglesias & 561 & 1.388 & $147,41 \%$ \\
& Miembros & $1 / 162$ & $1 / 80$ & - \\
\hline
\end{tabular}

64 Instituto Brasilero de Geografía y Estadística (IBGE), Secretaría de la Conferencia General de la Iglesia Adventista del Séptimo Día (IASD) y Secretaría de la Asociación Bahía Sul (ABS) de la IASD. Véase, Instituto Brasileiro de Geografia y Estadística (IBGE), "IBGE - Series Estadísticas \& Series Históricas"; General Conference of Seventh Day Adventist Church, "Annual Statistical Report for 1980"; General Conference of Seventh Day Adventist Church, "Annual Statistical Report for 1990", General Conference Archives, s.d., http://www. adventistarchives.org/doc_info.asp? DocID=118930 (consultado: 29 de julio, 2012); Secretaria da Associação Bahia Sul (ABS), Data da Fundação da Igreja, Quantidade de Igrejas e Membros até 31/12/2011 - Região de Eunápolis. 
Tabla 5. Crecimiento comparativo de la IASD en Brasil y en la zona metropolitana de Eunapolis (1990-2000) ${ }^{65}$

\begin{tabular}{|c|c|c|c|c|}
\hline LUGAR & AÑOS & 1990 & 2000 & $\%$ \\
\hline \multirow{4}{*}{ BRASIL } & Habitantes & 146.917 .459 & 169.590 .693 & $15,43 \%$ \\
& Iglesias & 5.334 & 8.728 & $63,62 \%$ \\
& Miembros & 541.186 & 993.876 & $83,64 \%$ \\
& Miembros/habitantes & $1 / 271$ & $1 / 171$ & - \\
\hline \multirow{3}{*}{ EUNAPOLIS } & Habitantes & 111.741 & 203.729 & $82,32 \%$ \\
& Iglesias & 17 & 37 & $117,64 \%$ \\
& Miembros & 1.388 & 2.550 & $83,71 \%$ \\
& Miembros/habitantes & $1 / 80$ & $1 / 80$ & - \\
\hline
\end{tabular}

Entre 1990 y 2000 , la población de Brasil pasó de 146.917.459 34 a 169.590 .693 habitantes, creciendo $15,43 \%$. En este mismo período, la población de Eunapolis pasó de 111.741 a 203.729 habitantes, creciendo $82,32 \%$. El número de iglesias adventistas en Brasil creció de 5.334 a 8.728 iglesias, es decir, hubo un incremento del 63,62\%. En la zona metropolitana de Eunapolis aumentó de 17 a 37, es decir, hubo

65 Instituto Brasilero de Geografía e Estadística (IBGE), Secretaría de la Conferencia General de la Iglesia Adventista del Séptimo Día (IASD) y Secretaría de la Asociación Bahía Sul (ABS) de la IASD. Véase, Ibíd. un incremento de $117,64 \%$. Los adventistas en Brasil aumentaron de 541.186 miembros a 993.876 , es decir, hubo un aumento de $83,64 \%$. En esta época, los adventistas en el área metropolitana de Eunapolis crecieron de 1.388 a 2.550 miembros, es decir, aumentaron en $83,71 \%$. Veáse la Tabla 5.

Por lo tanto, en la década de los 90, la tasa de crecimiento, en el número de iglesias del área metropolitana de Eunapolis, continuó mayor que la tasa de crecimiento de iglesias en Brasil. Pero, en número de miembros, el in- 
cremento fue aproximadamente igual a la población de la ciudad y de los miembros de la Iglesia adventista en el país, durante el mismo período.

Entre 2000 y 2010, la población de Brasil pasó de 169.590 .693 a 190.755 .799 habitantes, creciendo $12,48 \%$. En este mismo período, la población de Eunapolis pasó de 203.729 a 253.389 habitantes, creciendo $24,37 \%$. El número de Iglesias adventistas en Brasil creció de 8.728 a 13.610 iglesias, es decir, hubo un incremento del $55,93 \%$, y en la zona metropolitana de Eunapolis aumentó de 37 a 71, es decir, se incrementó en 91,89\%. Los adventistas en Brasil aumentaron de los 993.876 a 1.267 .738 miembros, es decir, aumentaron en $27,55 \%$. En esta época, los adventistas en el área metropolitana de Eunapolis crecieron de 2.550 a 6.705 miembros, es decir, aumentaron en 162,94\%. Veáse la Tabla 6.

Tabla 6. Crecimiento comparativo de la IASD en Brasil y en la zona metropolitana de Eunapolis (2000-2010). ${ }^{66}$

\begin{tabular}{|c|c|c|c|c|}
\hline LUGAR & AÑOS & 2000 & 2010 & $\%$ \\
\hline \multirow{3}{*}{ BRASIL } & Habitantes & 169.590 .693 & 190.755 .799 & $12,48 \%$ \\
& Iglesias & 8.728 & 13.610 & $55,93 \%$ \\
& Miembros & 993.876 & 1.267 .738 & $27,55 \%$ \\
& Miembros/habitantes & $1 / 171$ & $1 / 150$ & - \\
\hline \multirow{3}{*}{ EUNAPOLIS } & Habitantes & 203.729 & 253.389 & $24,37 \%$ \\
& Iglesias & 37 & 71 & $91,89 \%$ \\
& Miembros & 2.550 & 6.705 & $162,94 \%$ \\
& Miembros/habitantes & $1 / 80$ & $1 / 38$ & - \\
\hline
\end{tabular}

66 Instituto Brasilero de Geografía y Estadística (IBGE), Secretaría de la Conferencia General de la Iglesia Adventista del Séptimo Día (IASD) y Secretaría de la Asociación Bahía Sul (ABS) de la IASD. Véase, Ibíd. 
Por lo tanto, entre los años 1980 y 2010, la tasa de crecimiento en el número de iglesias y miembros en el área metropolitana de Eunapolis fue mayor que la tasa de crecimiento de la población en Brasil y de la Iglesia adventista en el país.

Sin embargo, si se considera desde 1978 cuando fue establecida la iglesia de ABBI hasta 2010, entonces el crecimiento del adventismo en la zona metropolitana de Eunapolis fue aún mayor que el mismo creci36 miento en el Brasil.

Entre 1978 y 2010, la población de Brasil pasó de 115.476 .085 a 190.755 .799 habitantes, creciendo 65,19\%. En este mismo período, la población de Eunapolis pasó de 88.101 a 253.389 habitantes, creciendo $187,61 \%$. El número de iglesias adventistas en Brasil creció de 2.565 a 13.610 iglesias, es decir, hubo un incremento del 430,60\%. En ese momento, el número de Iglesias adventistas, en la zona metropolitana de
Eunapolis, aumentó de 4 a 71, es decir, se incrementó en $1.675 \%$.

Por lo tanto, entre el año 1978, con el inicio del movimiento de plantío de iglesias y el año 2010, la tasa de crecimiento en el número de iglesias y miembros en el área metropolitana de Eunapolis fue significativamente mayor que la tasa de crecimiento de la población en Brasil y de la Iglesia adventista en el país, durante el mismo período.

\section{Conclusiones y recomendaciones}

1. La inmigración, ocurrida en la década de los setenta y noventa, transfirió para la región personas misioneras y emprendedoras, principalmente capixabas, que plantaron nuevas congregaciones en todo el área metropolitana de Eunapolis.

2. La Iglesia Adventista del Séptimo Día en Eunapolis, Bahía, Brasil, ha experimentado un in- 
cremento significativo del número de iglesias y miembros en los últimos años. Sin embargo, no se han encontrado estudios que describan este crecimiento.

3. La Iglesia central de Eunapolis fundó nuevas iglesias y estas fundaron otras a lo largo de la historia. Pero este fenómeno no se limita a una congregación, porque cuando ellas necesitan fundar una nueva iglesia, se unen y eso hace que el crecimiento se mantenga.

4. En el momento de la fundación de nuevos lugares, un número mínimo de hermanos se separan de sus iglesias. Ellos se unen, dejando por un tiempo sus iglesias, asisten, apoyan financieramente, predican y conquistan nuevos miembros. Entonces, regresan a sus iglesias de origen, y los nuevos miembros son los que desarrollan el cre- cimiento cuantitativo y organizan la nueva congregación.

5. Los cuatro principios bíblicos para el establecimiento de nuevas iglesias, según Rufus Anderson: ${ }^{67}$ 1. la conversión de los perdidos; 2. su organización en iglesias locales; 3 . promoción y formación de líderes en cada comunidad; 4. fomento de la independencia espiritual y de organización en cada comunidad, fueron verificadas en la estrategia de plantío de iglesias, en la zona metropolitana de Eunapolis. Tiempo y recursos fueron utilizados de modo que proporcionó resultados tangibles y coherentes con sus creencias y costumbres.

6. La estrategia de plantío de iglesias dio como resultado el incremento de feligreses adventistas

67 Anderson, Foreign Missions: Their Relations and Claims, 5; Beaver, "The Legacy of Rufus Anderson". 
en la región, evidenciado por el envejecimiento de la generación que estableció las primeras iglesias. La nueva generación ha conservado la estrategia, así, el crecimiento del adventismo es continuo en la región.

7. El adventismo en Eunapolis se ha expandido por medio de la estrategia del plantío de iglesias. Los feligreses adventistas creen que la estrategia del plantío de iglesias es la pieza clave por medio de la cual el mensaje adventista será transmitido al mundo.

8. Para ellos, la comisión del evangelio supone un establecimiento de iglesias agresivo y continuo, pues la estrategia del plantío de iglesias no es una moda; es un mandato, que viene de un amor genuino y radical que los mueve a plantar iglesias.

9. La estrategia de plantío de iglesias hace alcanzar a la creciente población por medio del establecimiento agresivo de nuevas iglesias. Como muchos perdidos ansían encontrar a Dios, las nuevas iglesias los ayudan.

10. Está bien establecido en Eunapolis que Dios ha colocado sobre todos los que creen, la responsabilidad de levantar iglesias, y no solamente sobre los pastores. Entonces, la estrategia del plantío de iglesias no es algo optativo: es un llamado y una misión.

11. Cuando un grupo de personas siente el llamado de Dios para plantar una nueva iglesia, las iglesias establecidas y los líderes encuentran la manera de alentar y animar, con recursos y personas, esta iniciativa.

12. En Eunapolis, la estrategia del plantío de iglesias es la manera normal de evangelizar, así la cantidad de miembros au- 
menta constantemente.

Para ellos, la recompensa de plantar iglesias nunca es monetaria, sino la felicidad de encontrarse con aquellos que conocieron a Dios en la iglesia plantada.

13. La estrategia del plantío de iglesias en Eunapolis está haciendo la diferencia. Rara vez las iglesias nuevas terminan siendo lo que los plantadores de iglesia esperaban; sino mucho más.

14. Las congregaciones que dan lo mejor de sí para establecer iglesias nuevas, se ven fortalecidas.

15. Las iglesias que han sido plantadas adecuadamente y estratégicamente alcanzaron a segmentos de la población que no ha- bían sido alcanzados por el mensaje adventista.

16. Plantar iglesias ha hecho surgir nuevos líderes, tanto en la iglesia madre como en la nueva iglesia.

17. El crecimiento cualitativo ocurrió implícitamente, como lo demuestra la actitud y participación de los feligreses, al invertir recursos financieros, tiempo y talentos en el plantío de nuevas iglesias.

18. Finalmente, en esta investigación, la estrategia del plantío de iglesias, sin duda alguna, contribuyó al incremento de feligreses adventistas en la zona metropolitana de Eunapolis, Bahía, Brasil, entre los años 1980 y 2010. 


\section{Bibliografía}

Abdala, Emílio. Guia de plantio de igrejas. Artur Nogueira, SP: UCB, 2010.

Bosch, David J. Missão transformadora: mudanças de paradigma na teologia da missão. São Leopoldo, RS: Editora Sinodal, 2002.

Dudley, Roger, and Clarence Gruesbeck. Plant a church, reap a harvest. Boise, ID: Pacific Press, 1989.

40 González, Justo L., and Carlos Cardoza Orlandi. História do movimento missionário. São Paulo, SP: Hagnos, 2010.

Hesselgrave, David J. Plantar igrejas. São Paulo: Vida Nova, 1988.

Hiebert, Paul G., and Eloise Hiebert Meneses. Incarnational Ministry: Planting churchs in band, tribal, pleasant and urban socie- ties. Grand Rapids, MI: Baker, 1995.

Lewis, Larry L. The church planter's handbook. Nashville, TN: Broadman, 1993.

Logan, Robert E. Beyond church growth. Old Tapan, NJ: Fleming H. Revell, 1989.

Malphurs, Aubrey. Planting growing churches for the 21 century: A comprehensive guide for new churches and those desiring revival. Grand Rapids, MI: Baker, 1998.

McIntosh, Gary L. Biblical church growth: How you can work with God to build a faithful church. Grand Rapids, MI: Baker, 2003.

Parker, Ken. Seven characteristics of a growing church. Pasadena, CA: Church Growth, 1979.

Peters, George W. A Theology of church growth. Grand Rapids, MI: Zondervan, 1981.

Rainer, Thom S. The book of church growth. Nashville, TN: BËH, 1998.

Wagner, C. Peter. Church planting for a greater harvest. Ventura, CA: Regal Books, 1990.

Warren, Rick. The purpose driven church: growth without compromising your message and mission. Grand Rapids, MI: Zondervan, 2007.

Waymire, Bob, and C. Peter Wagner. Manual de análisis de iglecrecimiento. 3 a ed. Santa Clara, CA: Global Church Growth, 1983.

White, Elena G. de. Cada día con Dios. Doral, FL: Publicaciones Interamericanas, 2003.

Zwetsch, Roberto E. "Missão - testemunho do evangelho no horizonte do reino de Deus". In Teologia Prática no contexto da América Latina, Editado por Cristoph Schneider-Harpprecht. São Paulo: ASTE, 2005. 